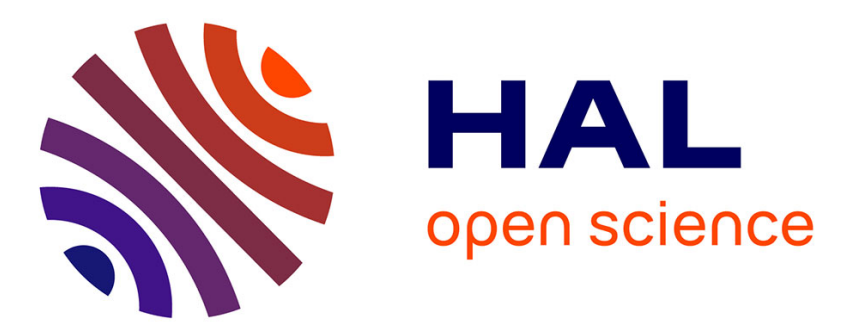

\title{
Random Euclidean Addition Chain Generation and Its Application to Point Multiplication
}

Fabien Herbaut, Pierre-Yvan Liardet, Nicolas Méloni, Yannick Teglia, Pascal Véron

\section{- To cite this version:}

Fabien Herbaut, Pierre-Yvan Liardet, Nicolas Méloni, Yannick Teglia, Pascal Véron. Random Euclidean Addition Chain Generation and Its Application to Point Multiplication. INDOCRYPT 2010, Dec 2010, Hyderabad, India. pp.238-261, 10.1007/978-3-642-17401-8_18 . hal-00674251

\section{HAL Id: hal-00674251 \\ https://hal.inria.fr/hal-00674251}

Submitted on 20 Mar 2012

HAL is a multi-disciplinary open access archive for the deposit and dissemination of scientific research documents, whether they are published or not. The documents may come from teaching and research institutions in France or abroad, or from public or private research centers.
L'archive ouverte pluridisciplinaire HAL, est destinée au dépôt et à la diffusion de documents scientifiques de niveau recherche, publiés ou non, émanant des établissements d'enseignement et de recherche français ou étrangers, des laboratoires publics ou privés. 


\title{
Random Euclidean Addition Chain Generation and Its Application to Point Multiplication
}

\author{
Fabien Herbaut ${ }^{1}$, Pierre-Yvan Liardet ${ }^{2}$, Nicolas Méloni ${ }^{3}$, \\ Yannick Téglia ${ }^{2}$, and Pascal Véron ${ }^{3}$ \\ 1 Université du Sud Toulon-Var, IMATH, France \\ IUFM de Nice, Université de Nice \\ herbaut@unice.fr \\ 2 ST Microelectronics, Rousset, France \\ pierre-yvan.liardet@st.com, yannick.teglia@st.com \\ 3 Université du Sud Toulon-Var, IMATH, France \\ meloni@univ-tln.fr, veron@univ-tln.fr
}

\begin{abstract}
Efficiency and security are the two main objectives of every elliptic curve scalar multiplication implementations. Many schemes have been proposed in order to speed up or secure its computation, usually thanks to efficient scalar representation [30 10 24], faster point operation formulae [8|25[13] or new curve shapes [2]. As an alternative to those general methods, authors have suggested to use scalar belonging to some subset with good computational properties [15/14/36 41/42], leading to faster but usually cryptographically weaker systems. In this paper, we use a similar approach. We propose to modify the key generation process using a small Euclidean addition chain $c$ instead of a scalar $k$. This allows us to use a previous scheme, secure against side channel attacks, but whose efficiency relies on the computation of small chains computing the scalar. We propose two different ways to generate short Euclidean addition chains and give a first theoretical analysis of the size and distribution of the obtained keys. We also propose a new scheme in the context of fixed base point scalar multiplication.
\end{abstract}

Keywords: point multiplication, exponentiation, addition chain, SPA, elliptic curves.

\section{Introduction}

After twenty five years of existence, elliptic curve cryptography (ECC) is now one of the major public-key cryptographic primitives. Its main advantages, compared to it main competitor RSA, are its shorter keys and the lack of fast theoretical attacks. The recent factorization of an RSA modulus of 768 bits 17 is here to highlight the significant role that ECC will play during the next decade. In particular, it has been shown that ECC is suitable for cryptographic applications on devices with small resources. However, if 160-bit ECC is believed to remain secure, from a theoretical point of view, at least until 2020 3, physical attacks on cryptographic devices have proved to be an immediate threat [22]. Thus,

G. Gong and K.C. Gupta (Eds.): INDOCRYPT 2010, LNCS 6498, pp. 238-261, 2010.

(C) Springer-Verlag Berlin Heidelberg 2010 
software or hardware ECC implementations have to deal with two apparently opposite requirements: efficiency and security. Indeed, protecting a device from physical attacks usually involves costly countermeasures.

In 2007, Méloni proposed a secure algorithm based on Euclidean addition chains [27. As they only involve additions, they are naturally resistant to SCA. However, the efficiency of such a method relies on the existence of a small chain computing the scalar. It has been pointed out that finding such a chain becomes more and more difficult when the scalar grows in size. For cryptographic sizes, finding a good chain is costlier than the scalar multiplication itself. So, instead of proposing a new scalar multiplication scheme, we propose to modify the key generation process. More precisely, we show that it is possible to generate the key as a small Euclidean addition chain, allowing us to use Méloni's fast and secure scheme.

From a general point of view, generating keys in a specific shape is not a new idea. Various methods have been proposed through the years to generate scalars belonging to some subset of the set of all possible keys, with good computational properties [14,36/41/42]. However, this usually implies some serious security issues 3334938 . Some methods remain cryptographically secure in the context of a fixed base point but require large amount of stored data [4]15] (Coron et al. 15 suggest to store from 50 to 100 points for the same security level as that considered in the present work). Finally, some schemes use special endomorphisms, such as the Frobenius map, on Koblitz curves [20].

From that perspective, our approach is quite different. Méloni's scheme only efficiently applies on curve in Weierstrass form. Moreover, it is particularly suitable to small devices with low computational resources because of its low memory requirement (at most two stored points) and resistance to side channel attacks. Yet, generating random Euclidean addition chains leads to several problems. First, what is the size of the keys we can generate for a given chain length? In other words, is it possible to achieve a certain level of security with relatively small chains? The second problem is that of distribution. Indeed, many different chains of same length can compute the same integer. It is then important to ensure that generating keys this way does not weaken the discrete logarithm problem.

In this paper, we produce the first practical and theoretical results on random Euclidean addition chain generation. We also show that it can lead to efficient and SPA-secure scalar multiplication methods.

This work is organized as follows. In Section 2 we review Méloni's scheme. In Section 3 we recall some background about Euclidean addition chains and set notations. In Section 4 and 5, we describe two different families of Euclidean addition chains and give some results on their distribution (notice that in Section 4 two variants are described). Finally, in Section 6 we propose some comparisons with existing side-channel resistant scalar multiplication methods.

\section{Scalar Multiplication Using Euclidean Addition Chains}

For the sake of concision, we do not give details about scalar multiplication and side channel attacks. We invite the reader to refer to [7/12 for detailed overview of elliptic curve based cryptography. 
This section is dedicated to a specific scalar multiplication algorithm based on Euclidean addition chains.

\subsection{Euclidean Addition Chains}

Definition 1. An Euclidean addition chain (EAC) of length $s$ is a sequence $\left(c_{i}\right)_{i=1 \ldots s}$ with $c_{i} \in\{0,1\}$. The integer $k$ computed from this sequence is obtained from the sequence $\left(v_{i}, u_{i}\right)_{i=0 . . s}$ such that $v_{0}=1, u_{0}=2$ and $\forall i \geqslant 1,\left(v_{i}, u_{i}\right)=$ $\left(v_{i-1}, v_{i-1}+u_{i-1}\right)$ if $c_{i}=1$ (small step), or $\left(v_{i}, u_{i}\right)=\left(u_{i-1}, v_{i-1}+u_{i-1}\right)$ if $c_{i}=0$ (big step). The integer $k$ associated to the sequence $\left(c_{i}\right)_{i=1 \ldots s}$ is $v_{s}+u_{s}$, we will denote it by $\chi(c)$.

ExAmPLE : From the EAC (10110) one can compute the integer 23 as follows : $(1,2) \rightarrow(1,3) \rightarrow(3,4) \rightarrow(3,7) \rightarrow(3,10) \rightarrow(10,13) \rightarrow 23=\chi(10110)$

\subsection{Point Multiplication Using EAC}

From any EAC $c$ and any point $P$ of an elliptic curve, it is shown in 27] that a new point $Q$ can be computed using the following algorithm :
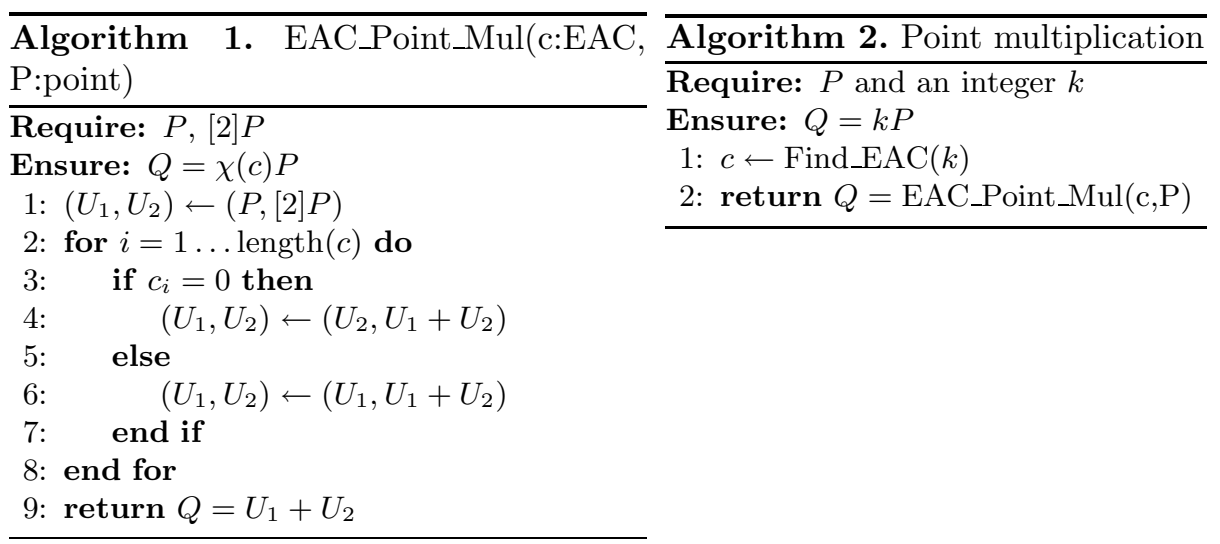

In [27, it is shown that any scalar multiplication can be performed using the preceding algorithm. It is achieved by finding an Euclidean addition chain computing the scalar. We will denote by Find_EAC, the algorithm which returns an addition chain for an integer $k$.

It has been shown in 27] that the for loop of algorithm 1 can be efficiently implemented on elliptic curves in Weierstrass form using Jacobian coordinates. This leads to a fast point multiplication method resistant to side channel attacks since at each step of the for loop, the same operation is used .

The efficiency of algorithm 2 directly depends on the length of the chains and the complexity of the algorithm Find_EAC. Although finding an Euclidean addition chain computing a given integer $k$ is quite simple (it suffices to choose an 
integer $g$ co-prime with $k$ and apply the subtractive form of Euclid's algorithm) finding a short chain remains a hard problem. As an example, the average length of the computed chain for $k$ with $g$ uniformly distributed in the range $[1, k]$ is $O\left(\ln ^{2} k\right)$ [18. For 160-bit scalars, experiments have shown that, on average, it is required to try more that 45,000 different $g$ to find a relatively small chain using the Montgomery heuristic 32 .

\subsection{Our Approach}

We propose in this paper to proceed differently. Instead of randomly choosing an integer $k$ and then trying to find a suitable EAC $c$ to finally compute the point $k P$, we propose to randomly generate a small length EAC $c$ and then compute the associated point on the curve. More precisely, we will see in the next sections that the chains will be chosen in a subset of short chains for key distribution matters.

From definition 2.1, notice the random generation of a $s$-length EAC boils down to the random generation of a $s$-bit integer. As an example, algorithm 3 shows how to process Diffie-Helmann key exchange protocol with EAC.

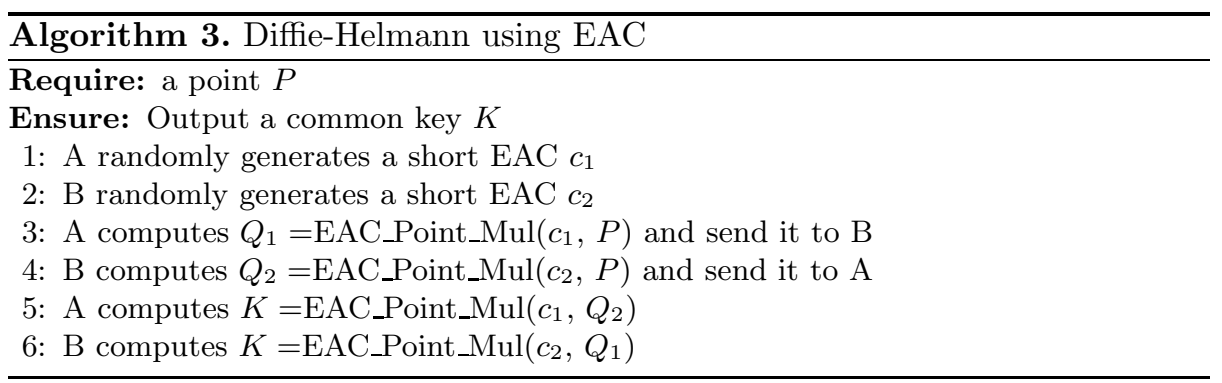

Using this approach we compute points $k P$ for a subset $S$ of all possible values for the integers $k$. Hence we do not deal any more with the classical Discrete Logarithm Problem but with the Constrained Discrete Logarithm Problem.

\footnotetext{
Name : CDLP

Input $\quad: p$ a prime number, $g$ a generator of a group $G, S \subset \mathbb{Z}_{p}$, and $g^{x}$ for $x \in S$.

Problem : Compute $x$.
}

The complexity of the discrete logarithm problem (DLP) over a generic group directly depends on the size of this group. It has been shown that the constrained version of this problem (CDLP), where only a subset $S$ of the group is considered, has a similar complexity. Indeed, for a random set $S, \Omega(\sqrt{|S|})$ group operations 28] are required to solve the CDLP. As an example, if one naively chooses to generate random Euclidean chain of length 160 (which means there are $2^{160}$ of them), we will see, in section 3 , that the biggest integer that can be generated is $F_{164} \simeq 2^{112.7}$. This means that the number of elements of the set $S$ is at most $2^{113}$, leading to a security of at most 66.5 bits. 
In this paper, we propose to study two families of EAC providing good balance between security and efficiency. In practice, it implies that the chains are long enough so that the corresponding set $S$ of generated keys is sufficiently large, but short enough so that the scalar multiplication remains fast.

\section{Notations and Properties}

We give in this section some notations and important results for the sequel of this paper.

Definition 2. Let $n$ and $p$ be two integers, we define :

. $\mathcal{M}$ as the set of $E A C$ and $\mathcal{M}_{n}$ as the set of $E A C$ of length $n>0$,

. $\mathcal{M}_{n, p}$ as the set of EAC of length $n>0$ and Hamming weight $p>0$.

- $\chi$ the map from $\mathcal{M}$ to $\mathbb{N}$, such that for $m \in \mathcal{M}, \chi(m)$ be the integer computed from the $E A C m$,

. $\psi$ the map from $\mathcal{M}$ to $\mathbb{N} \times \mathbb{N}$, such that for $m \in \mathcal{M}, \psi(m)=\left(v_{s}, u_{s}\right)$ if $m \in \mathcal{M}_{s}$,

. $S_{0}$ the matrix $\left(\begin{array}{ll}0 & 1 \\ 1 & 1\end{array}\right)$ corresponding to a big step iteration,

. $S_{1}$ the matrix $\left(\begin{array}{ll}1 & 1 \\ 0 & 1\end{array}\right)$ corresponding to a small step iteration.

With these notations, for $m=\left(m_{1}, \ldots, m_{s}\right) \in \mathcal{M}_{s}$, we have :

$$
\psi(m)=(1,2) \prod_{i=1}^{s} S_{m_{i}} \text { and } \chi(m)=\left\langle(1,2) \prod_{i=1}^{s} S_{m_{i}},(1,1)\right\rangle .
$$

Let $r$ and $s$ be two integers, we will denote by $\mathrm{mm}^{\prime}$ the element of $\mathcal{M}_{r+s}$ obtained from the concatenation of $m \in \mathcal{M}_{r}$ and $m^{\prime} \in \mathcal{M}_{s}$. This way, for $n>0, m^{n}$ is a word of $\mathcal{M}_{n r}$ if $m \in \mathcal{M}_{r}$.

For convenience, $\mathcal{M}_{0}$ will correspond to the set with one element $e$ which is the identity element for the concatenation.

For $m$ and $m^{\prime}$ two elements of $\mathcal{M}_{r}$ such that $\psi(m)=(v, u)$ and $\psi\left(m^{\prime}\right)=$ $\left(v^{\prime}, u^{\prime}\right)$ we will say that $\psi(m) \leqslant \psi\left(m^{\prime}\right)$ if $v \leqslant v^{\prime}$ and $u \leqslant u^{\prime}$.

Proposition 1. Let $n>0, F_{i}$ be the $i^{\text {th }}$ Fibonacci number, $\alpha_{n}=$ $\frac{(1+\sqrt{2})^{n}+(1-\sqrt{2})^{n}}{2}$ and $\beta_{n}=\frac{(1+\sqrt{2})^{n}-(1-\sqrt{2})^{n}}{2 \sqrt{2}}$ :

. $\psi\left(0^{n}\right)=\left(F_{n+2}, F_{n+3}\right), \psi\left(1^{n}\right)=(1, n+2), \chi\left(0^{n}\right)=F_{n+4}, \chi\left(1^{n}\right)=n+3$,

. $\forall m \in \mathcal{M}_{n}, \chi\left(1^{n}\right) \leqslant \chi(m) \leqslant \chi\left(0^{n}\right)$, and $\psi\left(1^{n}\right) \leqslant \psi(m) \leqslant \psi\left(0^{n}\right)$,

. $S_{0}^{n}=\left(\begin{array}{cc}F_{n-1} & F_{n} \\ F_{n} & F_{n+1}\end{array}\right), S_{1}^{n}=\left(\begin{array}{ll}1 & n \\ 0 & 1\end{array}\right)$,

$\left(S_{0} S_{1}\right)^{n}=\left(\begin{array}{cc}\alpha_{n}-\beta_{n} & \beta_{n} \\ \beta_{n} & \alpha_{n}+\beta_{n}\end{array}\right),\left(S_{1} S_{0}\right)^{n}=\left(\begin{array}{cc}\alpha_{n} & 2 \beta_{n} \\ \beta_{n} & \alpha_{n}\end{array}\right)$.

Proof. The first property is straightforward. The other ones can be proved by induction. 
Notice that from $S_{0}^{m+n}=S_{0}^{m} S_{0}^{n}$, we can recover a well known identity on Fibonacci sequence, namely :

$$
F_{m+n}=F_{m-1} F_{n}+F_{m} F_{n+1} .
$$

Proposition 2. Let $n>0$ and $m=\left(m_{1}, \ldots, m_{n}\right) \in \mathcal{M}_{n}$, then the map $\psi$ is injective and $\chi\left(m_{1}, \ldots, m_{n}\right)=\chi\left(m_{n}, \ldots, m_{1}\right)$.

Proof. We refer to [19] for standard link between EAC, Euclidean algorithm and continued fractions, which explains the second point. It is also explained that if $\psi(m)=(v, u)$ then $(u, v)=1$ and the only chain which leads to $(v, u)$ is obtained using the additive version of Euclidean algorithm.

\section{A First Family of EAC}

We will consider in this section $\mathcal{M}_{n}^{0}$ the subset of $\mathcal{M}_{2 n}$ whose elements are EAC beginning with $n$ zeros.

\subsection{Some Properties of $\mathcal{M}_{n}^{0}$}

From proposition 2 the restriction of $\chi$ to $\mathcal{M}_{n}$ is not injective because of the mirror symmetry property.

Proposition 3. The restriction of $\chi$ to $\mathcal{M}_{n}^{0}$ is injective.

Proof. Let $x$ and $y$ be two words of $\mathcal{M}_{n}^{0}$ such that $\chi(x)=\chi(y)$, and $m 0^{n}$, $m^{\prime} 0^{n}$, be the words obtained when reading $x$ and $y$ from right to left. Using the symmetry property, we have $\chi\left(m 0^{n}\right)=\chi\left(m^{\prime} 0^{n}\right)$. Let $(v, u)=\psi(m)$ and $\left(v^{\prime}, u^{\prime}\right)=\psi\left(m^{\prime}\right)$, then

$$
\begin{aligned}
\chi\left(m 0^{n}\right) & =\chi\left(m^{\prime} 0^{n}\right) \\
\Leftrightarrow F_{n} u+F_{n-1} v+F_{n+1} u+F_{n} v & =F_{n} u^{\prime}+F_{n-1} v^{\prime}+F_{n+1} u^{\prime}+F_{n} v^{\prime} \\
\Leftrightarrow \quad F_{n+2}\left(u-u^{\prime}\right) & =F_{n+1}\left(v^{\prime}-v\right)
\end{aligned}
$$

Since $\left(F_{n+1}, F_{n+2}\right)=1$, then $F_{n+2}$ divides $v^{\prime}-v$. Now from proposition 1 , since $v$ and $v^{\prime}$ are less or equal than $F_{n+2}$ and nonzero, then $\left|v^{\prime}-v\right|<F_{n+2}$ which implies that $v=v^{\prime}$ and so $u=u^{\prime}$. Hence $\psi(m)=\psi\left(m^{\prime}\right)$, so $m=m^{\prime}$.

Proposition 4. $\chi\left(\mathcal{M}_{n}^{0}\right) \subset\left[(n+1) F_{n+2}+F_{n+3}, F_{2 n+4}\right]$, the lower (resp. the upper) bound being reached by $0^{n} 1^{n}\left(\right.$ resp. $\left.0^{2 n}\right)$. The mean value is $\left(\frac{3}{2}\right)^{n} F_{n+4}$.

Proof. Let $0^{n} x 1 y$ and $0^{n} x 0 y$ be two elements of $\mathcal{M}_{n}^{0}$ where $x$ and $y$ are chains of size $a$ and $n-1-a$ with $a \in[0, n-1]$. From the definition of $\chi$ it follows that $\chi\left(0^{n} x 1 y\right)<\chi\left(0^{n} x 0 y\right)$. Hence the smallest integer is computed from the word $0^{n} 1^{n}$ and the greatest from $0^{n} 0^{n}$. Now $\chi\left(0^{n} 1^{n}\right)=\left\langle(1,2) S_{0}^{n} S_{1}^{n},(1,1)\right\rangle$ and $\chi\left(0^{2 n}\right)=\left\langle(1,2) S_{0}^{2 n},(1,1)\right\rangle$. From proposition 1 we deduce that $\chi\left(0^{n} 1^{n}\right)=(n+$ 1) $F_{n+2}+F_{n+3}$ and $\chi\left(0^{2 n}\right)=F_{2 n+4}$. 
To compute the mean value, let us consider $n$ independent Bernoulli random variables $C_{1}, \ldots, C_{n}$ such that $\forall i \in[1, n], \operatorname{Pr}\left(C_{i}=0\right)=\operatorname{Pr}\left(C_{1}=1\right)=1 / 2$. The mean value is $E(X)$ where $X=\chi\left(0^{n} C_{1} \ldots C_{n}\right)$. Now

$$
X=\left\langle(1,2) S_{0}^{n} \prod_{i=1}^{n}\left(\begin{array}{cc}
C_{i} & 1 \\
1-C_{i} & 1
\end{array}\right),(1,1)\right\rangle .
$$

Notice that $X$ is a polynomial of $\mathbb{Z}\left[C_{1}, \ldots, C_{n}\right] /\left(C_{1}^{2}-C_{1}, \ldots, C_{n}^{2}-C_{n}\right)$. As the $C_{i}$ are independent then $\forall J \subset[1, n], E\left(\prod_{j \in J} C_{j}\right)=\prod_{j \in J} E\left(C_{j}\right)$, hence

$$
E(X)=\left\langle(1,2) S_{0}^{n} \prod_{i=1}^{n}\left(\begin{array}{cc}
E\left(C_{i}\right) & 1 \\
1-E\left(C_{i}\right) & 1
\end{array}\right),(1,1)\right\rangle=\left\langle(1,2) S_{0}^{n} \prod_{i=1}^{n}\left(\begin{array}{cc}
\frac{1}{2} & 1 \\
\frac{1}{2} & 1
\end{array}\right),(1,1)\right\rangle .
$$

The final result comes from proposition 1 and the equality :

$$
\forall n \in \mathbb{N}^{*},\left(\begin{array}{cc}
\frac{1}{2} & 1 \\
\frac{1}{2} & 1
\end{array}\right)^{n}=(3 / 2)^{n-1}\left(\begin{array}{cc}
\frac{1}{2} & 1 \\
\frac{1}{2} & 1
\end{array}\right) .
$$

\subsection{Application to Existing Standards}

Using the set $\chi\left(\mathcal{M}_{n}^{0}\right)$, we can generate (with algorithm 1) $2^{n}$ distinct points $\chi(c) P$ for a point $P$ whose order is greater than $F_{2 n+4}$. Of course, when the order $d$ of the point $P$ is known, we have to choose the largest integer $n$ such that $F_{2 n+4}<d$.

Because of the results on the difficulty to solve the CDLP problem, we have to consider the set $\mathcal{M}_{n}^{0}$ only for $n \geqslant 160$. For $n=160$, we have $\chi\left(\mathcal{M}_{160}^{0}\right) \subset$ $\left[161 F_{162}+F_{163}, F_{324}\right]$, that is to say

$$
\chi\left(\mathcal{M}_{160}^{0}\right) \subset\left[2^{118.6}, 2^{223.6}\right] .
$$

Such data fit well with the secp224k1 and secp224r1 parameters where the order of the point $P$ is about $2^{223.99}$ 6. Those parameters are consistent with ANSI X.962, IEEE P1363 and IPSec standards and are recommended for ANSI X9.63 and NIST standards.

\subsection{A Variant for the secp160k1 and secp160r1 Recommended Parameters}

In the secp160k1 and secp160r 1 recommended parameters, the order $d$ of the point $P$ is around $2^{160}$. Using the set $\mathcal{M}_{n}^{0}$, this leads us to choose $n=114$ which only gives rise to $2^{114}$ distinct points. Hence the complexity of an attack is $\Omega\left(2^{57}\right)$ which may expose this method to some attacks.

Notice that if we use an element $c$ of $\mathcal{M}_{160}^{0}$ with the point $P$ of order $d$ then the algorithm 1 computes $(\chi(c) \bmod d) P$. If the values of $\{\chi(c) \bmod d, c \in$ $\left.\mathcal{M}_{160}^{0}\right\}$ are well distributed among $\mathbb{Z} / d \mathbb{Z}$, then we can use the above mentioned method, provided that computing $\chi(c) P$ with algorithm 1 be more efficient than computing $k P$ with $k \in \mathbb{Z} / d \mathbb{Z}$, with a classical SPA-resistant method. This last point will be discussed in section 6, we will focus now on the problem of the distribution. To this end, we will adapt results on Stern sequences from [35]. 
Table 1. Distribution of $\chi\left(\mathcal{M}_{n}^{0}\right)$ modulo $d$

\begin{tabular}{|c|c|c|c|c|c|c|c|c|c|c|c|c|c|c|c|}
\hline $\mathrm{n}$ & 0 & 1 & 2 & 3 & 4 & 5 & 6 & 7 & 8 & 9 & 10 & 11 & 12 & 13 & $\frac{\# \chi\left(\mathcal{M}_{0}^{n}\right)}{d}$ \\
\hline 10 & 294 & 312 & 214 & 70 & 12 & 4 & 1 & & & & & & & 0.66 \\
15 & 10814 & 12044 & 6216 & 2026 & 436 & 76 & 15 & & & & & & & 0.66 \\
20 & 266749 & 327372 & 194442 & 74219 & 20589 & 4344 & 789 & 100 & 18 & 1 & & & & 0.7 \\
25 & 6493638 & 8894037 & 5979946 & 2627531 & 850691 & 216285 & 44567 & 7832 & 1233 & 162 & 15 & 1 & 1 & 0.74 \\
29 & 74024780 & 115132679 & 88980442 & 45585634 & 17436296 & 5315191 & 1347286 & 294399 & 56344 & 9674 & 1459 & 193 & 18 & 4 & 0.79 \\
\hline
\end{tabular}

Theorem 1. Let $d$ be a prime number, $m \in \mathcal{M}_{\ell}$ such that $\psi(m)=(v, u)$. If $d \nmid u$, and $d \nmid v$ then there exist constants $c_{d} \in \mathbb{R}_{+}$and $\tau_{d} \in[0,1[$ so that for all $\alpha \in(\mathbb{Z} / d \mathbb{Z})^{*}, r \in \mathbb{N}$

$$
\begin{gathered}
\left|\frac{\#\left\{x \in \mathcal{M}_{r} \mid \chi_{d}(m x)=\alpha\right\}}{2^{r}}-\frac{d}{d^{2}-1}\right|<c_{d} \tau_{d}^{r}, \text { and } \\
\left|\frac{\#\left\{x \in \mathcal{M}_{r} \mid \chi_{d}(m x)=0\right\}}{2^{r}}-\frac{1}{d+1}\right|<c_{d} \tau_{d}^{r} .
\end{gathered}
$$

Proof. See annex for the proof and the link with Stern sequences.

Taking $m$ as the all zeros 160 bits vector, this asymptotic result let us think that the values $\chi(c)$ for $c \in \mathcal{M}_{160}^{0}$ are well distributed modulo $d$ since $\left(F_{162}, d\right)=$ $\left(F_{163}, d\right)=1$. In order to illustrate this theoretical result, we made several numerical tests by generating all EAC of $\mathcal{M}_{n}^{0}$ (from $n=10$ to $n=29$ ) and reducing the corresponding integer modulo a $n$-bits prime number (recall that for a practical use, we consider elements of $\mathcal{M}_{160}^{0}$ and a point $P$ of order $d$ about $2^{160}$ ). Table 1 seems to show that even for chains whose length is much smaller than $d$, the distribution is not so bad. For each value of $n$, a random $n$-bits prime $d_{n}$ has been generated. We have then computed for each $\alpha \in \mathbb{Z} / d_{n} \mathbb{Z}$, the cardinality $\delta_{\alpha}$ of $\chi^{-1}(\alpha)$ in $\mathcal{M}_{n}^{0}$. Let $\mathcal{T}=\left\{\delta_{\alpha} \mid \alpha \in \mathbb{Z} / d_{n} \mathbb{Z}\right\}$, for each $t$ in $\mathcal{T}$ we have then computed how many integers in $[0, d-1]$ are exactly computed $t$ times. As an example, for $t=0$ we know how many integers are never reached when reducing modulo $d$ the value $\chi(c)$ for $c \in \mathcal{M}_{n}^{0}$.

\section{A Second Family of EAC and an Open Problem}

In order to generate a 160 bits integer, the author of [26] gives numerical results which show that the search for a chain whose length be less than 260 needs about $2^{23}$ tests using a heuristic from Montgomery 32 . With such chains, Algorithm 1 needs less multiplications than the classical SPA-resistant algorithms. We investigated the problem of choosing shorter chains in order to speed up the performances of algorithm 1 . Once the length $\ell$ is fixed we have to deal with two constraints :

- the number $p$ of 1's in the chain must be chosen so that the greatest integer generated is as near as $d\left(\simeq 2^{160}\right)$ as possible, 
- because of the non-injectivity of $\chi,\left(\begin{array}{l}\ell \\ p\end{array}\right)$ must be greater than $2^{160}$ in order to hope that the integers generated reach most of the elements of $\left[1,2^{160}\right]$.

These two constraints lead us to study the sets $\mathcal{M}_{\ell, p}$ where $p<\ell / 2$.

Theorem 2. Let $(p, \ell) \in \mathbb{N}^{2}$ such that $0<2 p<\ell$. Let $F_{i}$ be the $i^{\text {th }}$ Fibonacci, $\alpha_{p}=\frac{(1+\sqrt{2})^{p}+(1-\sqrt{2})^{p}}{2}, \beta_{p}=\frac{(1+\sqrt{2})^{p}-(1-\sqrt{2})^{p}}{2 \sqrt{2}}$, then

i) For all $m \in \mathcal{M}_{\ell, p}$ we have, $F_{\ell-p+4}+p F_{\ell-p+2} \leqslant \chi(m) \leqslant F_{\ell-2 p+4}\left(\alpha_{p}+\beta_{p}\right)+$ $\beta_{p} F_{\ell-2 p+2}$.

ii) The lower bound is reached if and only if $m=1^{p} 0^{\ell-p}$ or $m=0^{\ell-p} 1^{p}$.

iii) The upper bound is reached if and only if $m=(01)^{p} 0^{\ell-2 p}$ or $m=0^{\ell-2 p}(10)^{p}$.

Proof. See annex.

To improve the performances of Algorithm 1, we choose to use chains whose length is 240 . In this case $p=80$ seems to be the best choice with respect to our two constraints. With such parameters, we can randomly generate about $2^{216}$ chains computing integers in the interval $\left[2^{117.7}, 2^{158.9}\right]$. Unfortunately, it seems to be a hard problem to compute the number of distinct integers generated in this way.

This naturally leads us to consider the set $\mathcal{M}_{3 p, p}$. Numerical experiments for some values of $p$ let us think that the cardinality of $\chi\left(\mathcal{M}_{3 p, p}\right)$ is near from $2^{2 p}$. Notice that from the preceding theorem, it can be proved that the upper bound for $\ell=3 p$ is equivalent to $\gamma\left(\frac{(1+\sqrt{2})(1+\sqrt{5})}{2}\right)^{p}$ where $\gamma=\frac{(1+\sqrt{5})^{4}}{32 \sqrt{5}}+\frac{(1+\sqrt{5})^{4}}{32 \sqrt{10}}+$ $\frac{(1+\sqrt{5})^{2}}{8 \sqrt{10}}$ which is close to $\gamma 2^{1.96 p}$. We end this section with an open problem : What is the cardinality of $\chi\left(\mathcal{M}_{3 p, p}\right)$ ? The good performances of this method (see next section) make this problem of interest. Notice that a straightforward argument gives that the number of distinct integers generated (for the proposed parameters) is greater than $2^{106}$. Indeed, it follows from proposition 3 that the chains $0^{120} c$, where $c \in \mathcal{M}_{120,80}$ give rise to distinct integers.

\section{Comparisons}

In this paper we have proposed three different ways to compute a point on the curve from an Euclidean addition chain :

Method 1: use a chain from $\mathcal{M}_{160}^{0}$ for curves of order about $2^{224}$.

Method 2: use a chain from $\mathcal{M}_{160}^{0}$ for curves of order about $2^{160}$ even if $\chi(c)$ can be greater than the order, using the results on Stern sequences.

Method 3: use a chain from $\mathcal{M}_{240,80}$ for curves of order about $2^{160}$.

The interest of Method 1 is that it is the only method for which we have a proved security. The main drawback is that it forces us to work on curves defined over larger fields $\left(\simeq 2^{224}\right.$ elements instead of $\left.2^{160}\right)$. However, we will see, 
in Section 6.2, that it might not be such a problem in practical implementations. Moreover, this method shows to be particularly relevant in the context of fixed base point scalar multiplication.

Method 2 allows us to reduce the size of the underlying fields by using Stern's results. Numerical samples tend to show that the generated keys are well distributed but we still lack a complete security proof.

Finally, in Method 3, we try to reduce both the size of the fields and the length of the chains. In that case, it becomes very complicated to analyze the distribution of the generated keys. In particular, the system becomes highly redundant, which might lead to a bias in the set of possible chains. Typically, this would make this method irrelevant for signature and key-exchange schemes.

We propose to compare our work to other side-channel resistant methods with a similar security level. We do not take into account special key generation methods as they usually provide lower security level 33.34. One could try to increase the size of the underlying field (as we do with Method 1) to solve this issue, however this would make those approaches slower than general algorithms. In the fixed point scenario, special key generation methods usually provide enough security but require in the same time a large amount of stored data: from 50 to 1000 precomputed points [154] when we only require a table of two stored points.

In Table 2, we summarize the cost, in terms of field multiplications, of various SPA-resistant scalar multiplication schemes providing a security of 80 bits. In order to ease comparisons, we make the traditional assumption that the cost of a field squaring $(\mathrm{S})$ is 80 percent of that of a field multiplication (M).

Random Base Point. In that scenario, a new base point $P$ is computed for each new session. This implies that it is not possible to precompute offline multiples of $P$ to speed up the process.

We consider the following SPA resistant methods:

- Dummy operations consist of adding a dummy point addition during the double-and-add algorithm, when the current bit is a 0 .

- The Montgomery ladder is a SPA resistant algorithm from Peter Montgomery [31, performing one doubling and one addition for each bit of the scalar. It is only efficient on Montgomery curves.

- Unified formulae allow to perform doubling and addition with the same formulae on specific curve shapes. They can be then combined with the NAF representation for scalar multiplication. The cost of the unified operation is 11M, 12M and 14M on Edwards [2], Hessian [16] and Jacobi curves [23] respectively. We do not compare to Brier and Joye general unified formulae due to their quite high cost (18M) [5].

- Möller proposed a modified version of Brauer ( $2^{w}$-ary) algorithm [29]. Using precomputations, its pattern is independent from the scalar itself. In that case, we used the latest and fastest point addition and point doubling formulae in Jacobian coordinates (see [1] for complete overview). One can also refer to the Left-to-Right recodings methods proposed in [39] whose performances are similar. 
Fixed Base Point. In the context of a fixed base point we propose a new scalar multiplication scheme based on our chains generation method. Notice that in Methods 1 and 2, the 160 first steps of Algorithm 1 are fixed, independently of the scalar, and correspond to big steps. Hence we can precompute and store the points $F_{162} P$ and $F_{163} P$ and then generate random chains of length 160 . We compared these methods to the classical Comb method.

Table 2. Cost of various SPA resistant scalar multiplication methods providing 80 bits of security

\begin{tabular}{|c|c|c|c|c|c|}
\hline Point gen. & Method & curve & field size (bits) & \# precomp. points & \#Field Mult. \\
\hline \hline \multirow{5}{*}{ random } & Dummy operations & general & 160 & 1 & 3530 \\
& Montgomery ladder & Montgomery & 160 & 1 & 1463 \\
& Unified formulae & Edwards & 160 & 1 & 2335 \\
& Unified formulae & Hessian & 160 & 1 & 2548 \\
& Unified formulae & Jacobi & 160 & 1 & 2973 \\
& Unified formulae & Edwards & 160 & 7 & 2130 \\
& Unified formulae & Hessian & 160 & 7 & 2324 \\
& Unified formulae & Jacobi & 160 & 7 & 2711 \\
& Möller's recoding & general & 160 & 16 & 1843 \\
\hline \hline \multirow{5}{*}{ fixed } & Comb Method & general & 160 & 2 & 1754 \\
& Comb Method & general & 160 & 4 & 1177 \\
& Comb Method & general & 160 & 8 & 866 \\
& Comb Method & general & 160 & 16 & 688 \\
\hline \hline \multirow{2}{*}{ random } & Method 1 & general & 224 & 1 & $\mathbf{2 1 0 4}$ \\
& Method $1(x$ only $)$ & general & 224 & 1 & $\mathbf{1 7 9 0}$ \\
\hline \multirow{2}{*}{ fixed } & Method 1 & general & 224 & 2 & $\mathbf{1 0 4 8}$ \\
& Method $1(x$ only $)$ & general & 224 & 2 & $\mathbf{8 8 8}$ \\
\hline \hline \multirow{2}{*}{ random } & Method 2 & general & 160 & 1 & $\mathbf{2 1 0 4}$ \\
& Method $2(x$ only $)$ & general & 160 & 1 & $\mathbf{1 7 9 0}$ \\
& Method 3 & general & 160 & 1 & $\mathbf{1 5 7 6}$ \\
& Method 3 $(x$ only $)$ & general & 160 & 1 & $\mathbf{1 3 3 6}$ \\
\hline \multirow{2}{*}{ fixed } & Method 2 & general & 160 & 2 & $\mathbf{1 0 4 8}$ \\
& Method 2 $(x$ only $)$ & general & 160 & 2 & $\mathbf{8 8 8}$ \\
\hline
\end{tabular}

To be completely fair, we evaluate in the next section the additional cost of working on larger fields with Method 1.

As for Method 2 and 3, Table 2 shows our different methods provide very good results. In the random point scenario, Methods 2 and 3 perform generally better that their counterparts. Only Montgomery's algorithm can be claim to be faster, but its use is restricted to Montgomery's curves. Besides, computing the $x$ coordinate only with Method 3 leads to a faster scheme. In the fixed based point scenario, the Comb method requires at least 4 times more stored points to perform faster than our methods. 


\subsection{Comparison of Method 1 with Algorithms Working on 160-Bit Integers}

Recall that Method 1 requires to work on fields of larger size ( $2^{224}$ elements). Hence to be fair in our comparisons, we need to evaluate the additional cost of performing multiplications in larger fields. To this end, we are going to consider three contexts for modular multiplication. For each of them, we will identify scenarios for which it is worth using our method.

a) The CIOS method [21]: the Coarsely Integrated Operand Scanning method is an efficient implementation of Montgomery's modular multiplication for a large class of processor. From [21] a modular multiplication between two integers stored as $s$ words of $w$ bits needs $2 s^{2}+s w$-bits multiplications, $4 s^{2}+4 s+2$ $w$-bits additions, $6 s^{2}+7 s+2 w$-bits read instructions and $2 s^{2}+5 s+1 w$-bits write instructions. Using these results, we can estimate the cost in terms of $w$ bits operations of the methods listed in Table 2. This leads us to the following remarks.

On a 32 bits processor, for a random point $P$, Method 1 with $x$-only is the most performant SPA resistant method which can be used on any curve and which only stores the point $P$. For a fixed point $P$, if only two points can be stored, this latter is better than Comb method. On a 64 bits processor, the preceding remarks remain true. Moreover, the fixed point method (without the $x$ coordinate trick) is competitive with the Comb method when only two points are stored. On a 128 bits processor, since two words are needed to store 160 bits integer or 224 bits integer, we only have to compare the number of field multiplications in Table 2. This shows that our method in the fixed point context is better than the Comb method when storing 2 or 4 points. For random point context, if one needs an SPA resistant algorithm which works on any curve and stores no more than one point, then our method gives the best result.

b) The GNU multiprecision library: we provide benchmarks for fair comparisons between modular multiplications in the case of practical use with the library GMP. We compute several times $2^{28}$ modular multiplications (using

Table 3. Performances of Method 1 using CIOS method on 32 bits processor

\begin{tabular}{|c|c|c|c|c|c|c|}
\hline & S & \# Field mult. & 32 bits $\times$ & 32 bits + & 32 bits Read & 32 bits Write \\
\hline Method 1, $x$-only & 7 & 1790 & 187950 & 404540 & 617550 & 239860 \\
Dummy & 5 & 3530 & 194150 & 430660 & 660110 & 268280 \\
\hline Method 1, fixed point, $x$-only & 7 & 888 & 93240 & 200688 & 306360 & 118992 \\
Comb method, 2 stored points & 5 & 1754 & 96470 & 213988 & 327998 & 133304 \\
\hline
\end{tabular}

Table 4. Performances of Method 1 using CIOS method on 64 bits processor

\begin{tabular}{|c|c|c|c|c|c|c|}
\hline & $\mathrm{s}$ & \# Field mult. & 64 bits $\times$ & 64 bits + & 64 bits Read & 64 bits Write \\
\hline Method 1, fixed point & 4 & 1048 & 37728 & 85936 & 132048 & 55544 \\
\hline Comb method, 2 stored points & 3 & 1754 & 36834 & 87700 & 135058 & 59636 \\
\hline
\end{tabular}


Table 5. Time to compute $2^{28}$ modular multiplications with GnuMP

\begin{tabular}{|c|c|c|c|}
\hline & $p$ & 32 bits Intel T2500 2.0Ghz & 64 bits AMD Opteron $83822.6 \mathrm{Ghz}$ \\
\hline \multirow{2}{*}{$160 \mathrm{bits}$} & $2^{160}-2^{31}-1$ & $T_{\min } 135.96 \mathrm{~s}$ & $T_{\min } 28.12 \mathrm{~s}$ \\
& & $T_{\max } 140.49 \mathrm{~s}$ & $T_{\max } 32.49 \mathrm{~s}$ \\
& $T_{\text {average }} 137.88 \mathrm{~s}$ & $T_{\text {average }} 30.42 \mathrm{~s}$ \\
\hline \multirow{2}{*}{$224 \mathrm{bits}$} & \multirow{2}{*}{$2^{224}-2^{96}+1$} & $T_{\min } 198.22 \mathrm{~s}$ & $T_{\min } 32.24 \mathrm{~s}$ \\
& & $T_{\max } 201.86 \mathrm{~s}$ & $T_{\max } 33.93 \mathrm{~s}$ \\
\hline$T_{\text {average 224 }} / T_{\text {average 160 }}$ & $T_{\text {average }} 200.51 \mathrm{~s}$ & $T_{\text {average }} 32.67 \mathrm{~s}$ \\
$T_{\max 224} / T_{\min 160}$ & 1.45 & 1.07 \\
\hline
\end{tabular}

Table 6. Performances of Method 1 with GnuMP

\begin{tabular}{|c|c|c|c|c|c|}
\hline Point gen. & Method & curve & storage & Mult. (32 bits proc.) & Mult. (64 bits proc.) \\
\hline \hline \multirow{2}{*}{ random } & Method 1 & general & 1 & 3114 & 2462 \\
& Method 1 $(x$ only) & general & 1 & 2650 & 2095 \\
\hline \multirow{2}{*}{ fixed } & Method 1 & general & 2 & 1552 & 1227 \\
& Method 1 $(x$ only) & general & 2 & 1315 & 1039 \\
\hline
\end{tabular}

mpz_mul and mpz_mod) on 32 and 64 bits processors. We consider reduction modulo a prime number $p$ conformant to the ANSI X9.63 standard 6] (resp. FIPS186-3 standard [40]) for the 160 bits (resp. 224 bits) case. From these benchmarks, we deduce an average time to compute a modular multiplication as detailed in table 5. Let us now consider the ratio in the most pessimistic case : the cost of a 224 bits modular multiplication is 1.17 times (resp. 1.48 times) the cost of a 160 bits multiplication for 64 bits (resp. 32 bits) processor. Taking into account these results, we can give an estimate in terms of $\mathbf{1 6 0}$ bits multiplications of Method 1. This shows the interest of Method 1, specially in the fixed point context (see table 2).

c) Hardware context: we considered in this section two kinds of components from the STMicroelectronics portfolio. The first embeds the Public Key 64 bits crypto processor from AST working at 200Mhz (CORE65LPHVT technology) and the second the 128 bits hardware smartcard cryptographic coprocessor Nescrypt working at $110 \mathrm{Mhz}$. The AST crypto processor can compute about 2040816 160-bits modular multiplications and 1449275 224-bits modular multiplications per second. Taking into account these results, table 7 gives the time needed by the modular multiplications when computing a point multiplication. Once again, in the random point context, Method 1 obtains best performances if one needs an SPA resistant algorithm working on a general curve and storing only one point. In the fixed point context, Method 1 is faster than the Comb method with two points. Notice that we only consider the multiplications done by the cryptoprocessor for the times given in table 7 . We do not take into account the overhead involved by the communications between the processor and the crypto processor. 
Table 7. Time comparison for scalar multiplication methods in milliseconds

\begin{tabular}{|c|c|c|c|c|}
\hline Point gen. & Method & curve & \# precomp. points & msecs \\
\hline \hline & Dummy operations & general & 1 & 1.73 \\
& Montgomery ladder & Montgomery & 1 & 0.717 \\
& Unified formulae & Edwards & 1 & 1.144 \\
& Unified formulae & Hessian & 1 & 1.249 \\
random (160 bits) & Unified formulae & Jacobi & 1 & 1.457 \\
& Unified formulae & Edwards & 7 & 1.044 \\
& Unified formulae & Hessian & 7 & 1.139 \\
& Unified formulae & Jacobi & 7 & 1.328 \\
& Möller's recoding & general & 16 & 0.903 \\
\hline \hline \multirow{5}{*}{ fixed (160 bits) } & Comb Method & general & 2 & 0.859 \\
& Comb Method & general & 4 & 0.577 \\
& Comb Method & general & 8 & 0.424 \\
& Comb Method & general & 16 & 0.337 \\
\hline \hline \multirow{2}{*}{ random (224 bits) $)$} & Method 1 & general & 1 & $\mathbf{1 . 4 5 2}$ \\
& Method 1 $(x$ only) & general & 1 & $\mathbf{1 . 2 3 5}$ \\
\hline \multirow{2}{*}{ fixed (224 bits) } & Method 1 & general & 2 & $\mathbf{0 . 7 2 3}$ \\
& Method 1 $(x$ only) & general & 2 & $\mathbf{0 . 6 1 3}$ \\
\hline
\end{tabular}

Nescrypt 128 bits crypto processor can compute about 339506 modular multiplications per second both for 160 bits and 224 bits integers. Indeed in both cases, only two 128 bits blocks are used to manipulate these integers. Hence we can do the same remarks as in the section about the CIOS method.

\section{Conclusions}

The goal of this paper was to describe subsets of integers $k$ for which the computation of $k P$ is faster, when dealing with the problem of $S P A$-secure exponentiation over an elliptic curve. We studied three such subsets and produced the first practical and theoretical results on random Euclidean addition chain generation. Table 2 shows that our methods provide good results in various situations when compared with the best SPA-secure methods.

We proved that the Method 1 we considered is secure and fast. In particular, in the context of a fixed base point, it is competitive with actual methods and faster when using similar amount of storage. We detailed several practical scenarios for which the method is relevant and improves efficiency : in CIOS context, in the context of GNU multiprecision library, and on some cryptoprocessors.

At last, we began the theoretical study of the other proposed methods. We made links between Method 2 and Stern sequences which enabled to obtain optimistic but asymptotic results. We managed to begin the study of the distribution of integers generated by Method 3. Both methods would improve the performances once again. For example Method 3 would be faster than Montgomery ladder, thus it would be worth studying it further. We have proved some results that may be useful for any further investigation. 


\section{References}

1. Bernstein, D.J., Lange, T.: Explicit-formulas database, http://hyperelliptic.org/EFD

2. Bernstein, D.J., Lange, T.: Faster addition and doubling on elliptic curves. In: Kurosawa, K. (ed.) ASIACRYPT 2007. LNCS, vol. 4833, pp. 29-50. Springer, Heidelberg (2007)

3. Bos, J.W., Kaihara, M.E., Kleinjung, T., Lenstra, A.K., Montgomery, P.L.: On the security of 1024-bit rsa and 160-bit elliptic curve cryptography. Technical report, EPFL IC LACAL and Alcatel-Lucent Bell Laboratories and Microsoft Research (2009)

4. Boyko, V., Peinado, M., Venkatesan, R.: Speeding up discrete log and factoring based schemes via precomputations (1998)

5. Brier, E., Joye, M.: Weierstraß elliptic curves and side-channel attacks. In: Naccache, D., Paillier, P. (eds.) PKC 2002. LNCS, vol. 2274, pp. 335-345. Springer, Heidelberg (2002)

6. Certicom Research. Sec 2: Recommended elliptic curve domain parameters standards for efficient cryptography. Technical report, Certicom (2000)

7. Cohen, H., Frey, G., Avanzi, R., Doche, C., Lange, T., Nguyen, K., Vercauteren, F.: Handbook of Elliptic and Hyperelliptic Cryptography, Discrete Mathematics and its Applications, vol. 34. Chapman \& Hall/CRC (2005)

8. Cohen, H., Miyaji, A., Ono, T.: Efficient elliptic curve exponentiation using mixed coordinates. In: Ohta, K., Pei, D. (eds.) ASIACRYPT 1998. LNCS, vol. 1514, pp. 51-65. Springer, Heidelberg (1998)

9. de Rooij, P.: On Schnorr's preprocessing for digital signature schemes. In: Helleseth, T. (ed.) EUROCRYPT 1993. LNCS, vol. 765, pp. 435-439. Springer, Heidelberg (1994)

10. Dimitrov, V., Imbert, L., Mishra, P.K.: Efficient and secure elliptic curve point multiplication using double-base chains. In: Roy, B. (ed.) ASIACRYPT 2005. LNCS, vol. 3788, pp. 59-78. Springer, Heidelberg (2005)

11. Graham, R.L., Knuth, D.E., Patashnik, O.: Concrete Mathematics: A Foundation for Computer Science. Addison-Wesley, Reading (1989)

12. Hankerson, D., Menezes, A., Vanstone, S.: Guide to Elliptic Curve Cryptography. Springer, Heidelberg (2004)

13. Hisil, H., Koon-Ho Wong, K., Carter, G., Dawson, E.: Twisted Edwards curves revisited. In: Pieprzyk, J. (ed.) ASIACRYPT 2008. LNCS, vol. 5350, pp. 326-343. Springer, Heidelberg (2008)

14. Hoffstein, J., Silverman, J.H.: Random small hamming weight products with applications to cryptography. Discrete Appl. Math. 130(1), 37-49 (2003)

15. M'Raïhi, D., Coron, J.-S., Tymen, C.: Fast generation of pairs (k, [k]p) for koblitz elliptic curves. In: Vaudenay, S., Youssef, A.M. (eds.) SAC 2001. LNCS, vol. 2259, pp. 151-174. Springer, Heidelberg (2001)

16. Joye, M., Quisquater, J.-J.: Hessian elliptic curves and side-channel attacks. In: Koç, Ç.K., Naccache, D., Paar, C. (eds.) CHES 2001. LNCS, vol. 2162, pp. 402410. Springer, Heidelberg (2001)

17. Kleinjung, T., Aoki, K., Franke, J., Lenstra, A.K., Thomé, E., Bos, J.W., Gaudry, P., Kruppa, A., Montgomery, P.L., Osvik, D.A., te Riele, H., Timofeev, A., Zimmermann, P.: Factorization of a 768-bit rsa modulus. Technical report, EPFL IC LACAL and NTT and University of Bonn and INRIA CNRS LORIA and Microsoft Research and CWI (2010) 
18. Knuth, D., Yao, A.: Analysis of the subtractive algorithm for greater common divisors. Proc. Nat. Acad. Sci. USA 72(12), 4720-4722 (1975)

19. Knuth, D.E.: The Art of Computer Programming: Fundamental Algorithms, 3rd edn, vol. 2. Addison Wesley, Reading (July 1997)

20. Koblitz, N.: CM-curves with good cryptographic properties. In: Feigenbaum, J. (ed.) CRYPTO 1991. LNCS, vol. 576, pp. 279-287. Springer, Heidelberg (1992)

21. Koc, C.K., Acar, T.: Analyzing and comparing montgomery multiplication algorithms. IEEE Micro 16, 26-33 (1996)

22. Kocher, P., Jaffe, J., Jun, B.: Differential power analysis. In: Wiener, M. (ed.) CRYPTO 1999. LNCS, vol. 1666, pp. 388-397. Springer, Heidelberg (1999)

23. Liardet, P.-Y., Smart, N.P.: Preventing SPA/DPA in ECC systems using the Jacobi form. In: Koç, Ç.K., Naccache, D., Paar, C. (eds.) CHES 2001. LNCS, vol. 2162, pp. 391-401. Springer, Heidelberg (2001)

24. Longa, P., Gebotys, C.: Setting speed records with the (fractional) multibase nonadjacent form method for efficient elliptic curve scalar multiplication. In: Jarecki, S., Tsudik, G. (eds.) Public Key Cryptography - PKC 2009. LNCS, vol. 5443, pp. 443-462. Springer, Heidelberg (2009)

25. Longa, P., Miri, A.: New composite operations and precomputation scheme for elliptic curve cryptosystems over prime fields. In: Cramer, R. (ed.) PKC 2008. LNCS, vol. 4939, pp. 229-247. Springer, Heidelberg (2008)

26. Meloni, N.: Arithmétique pour la Cryptographie basée sur les Courbes Elliptiques. $\mathrm{PhD}$ thesis, Université de Montpellier, France (2007)

27. Meloni, N.: New point addition formulae for ECC applications. In: Carlet, C., Sunar, B. (eds.) WAIFI 2007. LNCS, vol. 4547, pp. 189-201. Springer, Heidelberg (2007)

28. Mironov, I., Mityagin, A., Nissim, K.: Hard instances of the constrained discrete logarithm problem. In: Hess, F., Pauli, S., Pohst, M. (eds.) ANTS 2006. LNCS, vol. 4076, pp. 582-598. Springer, Heidelberg (2006)

29. Möller, B.: Securing elliptic curve point multiplication against side-channel attacks. In: Davida, G.I., Frankel, Y. (eds.) ISC 2001. LNCS, vol. 2200, pp. 324-334. Springer, Heidelberg (2001)

30. Möller, B.: Improved techniques for fast exponentiation. In: Lee, P.J., Lim, C.H. (eds.) ICISC 2002. LNCS, vol. 2587, pp. 298-312. Springer, Heidelberg (2003)

31. Montgomery, P.: Speeding the pollard and elliptic curve methods of factorization. Mathematics of Computation 48, 243-264 (1987)

32. Montgomery, P.L.: Evaluating recurrences of form $X_{m+n}=f\left(X_{m}, X_{n}, X_{m-n}\right)$ via Lucas chains (1992), http://ftp.cwi.nl/pub/pmontgom/Lucas.ps.gz

33. Mui, J.A., Stinson, D.R.: On the low hamming weight discrete logarithm problem for nonadjacent representations. Applicable Algebra in Engineering, Communication and Computing 16, 461-472 (2006)

34. Nguyên, P.Q., Stern, J.: The hardness of the hidden subset sum problem and its cryptographic implications. In: Wiener, M. (ed.) CRYPTO 1999. LNCS, vol. 1666, pp. 31-46. Springer, Heidelberg (1999)

35. Reznick, B.: Regularity properties of the stern enumeration of the rationals. Journal of Integer Sequences 11 (2008)

36. Schnorr, C.P.: Efficient signature generation by smart cards. Journal of Cryptology 4, 161-174 (1991)

37. Stern, M.A.: über eine zahlentheoretische funktion. Journal für die reine und angewandte Mathematik 55, 193-220 (1858)

38. Stinson, D.R.: Some baby-step giant-step algorithms for the low hamming weight discrete logarithm problem. Mathematics of Computation 71, 379-391 (2000) 
39. Theriault, N.: Spa resistant left-to-right integer recodings. In: Preneel, B., Tavares, S. (eds.) SAC 2005. LNCS, vol. 3897, pp. 345-358. Springer, Heidelberg (2006)

40. U.S. Department of Commerce and National Intitute of Standards and Technology. Digital signature standard (DSS). Technical report (2009)

41. Yacobi, Y.: Exponentiating faster with addition chains. In: Damgård, I.B. (ed.) EUROCRYPT 1990. LNCS, vol. 473, pp. 222-229. Springer, Heidelberg (1991)

42. Yacobi, Y.: Fast exponentiation using data compression. SIAM J. Comput. 28(2), 700-703 (1999)

\section{Annex}

\section{Proof of Theorem 1}

Definition 3. Let $(a, b) \in \mathbb{N}^{2}$, the generalized Stern sequence $\left(s_{a, b}(r, n)\right)_{r \in \mathbb{N}, n \in\left[0,2^{r}\right]}$ is defined by $s_{a, b}(0,0)=a, s_{a, b}(0,1)=b$, and for $r \geqslant 1$, $s_{a, b}(r, 2 n)=s_{a, b}(r-1, n), s_{a, b}(r, 2 n+1)=s_{a, b}(r-1, n)+s_{a, b}(r-1, n+1)$.

In his original paper, Stern gave a practical description of his sequence using the following diatomic array [37] :

$$
\begin{aligned}
& (r=0) \quad a \quad b \\
& (r=1) a a+b \quad b \\
& (r=2) a 2 a+b a+b \quad a+2 b \quad b \\
& (r=3) a 3 a+b 2 a+b 3 a+2 b a+b 2 a+3 b a+2 b a+3 b b
\end{aligned}
$$

where each line $r$ is exactly the sequence $s_{a, b}(r, n)$ for $n \in\left[0,2^{r}\right]$. Notice that to compute the row $r$, you just have to rewrite row $r-1$ and insert their sum between two elements. In the case $(a, b)=(1,1)$, the sequence is called the Stern sequence and has been well studied. For example, see the introduction of [35] or 11] for the link with the Stern Brocot array.

Now we will point deep connections between Stern sequences and the $\psi$ and $\chi$ maps. These connections should not surprise us, because both are linked with continued fractions. As an example, if $(a, b)=(1,1)$, an easy induction enables us to prove that when $n \in\left[0,2^{r}\right.$, the sequence $\left(s_{1,1}(r+1,2 n), s_{1,1}(r+1,2 n+1)\right)$ describes the set $\psi\left(\mathcal{M}_{r}\right)$.

$$
\begin{aligned}
& (r=0) 11 \\
& (r=1) 121 \\
& (r=2) 13231 \\
& (r=3) 143525341 \quad\left(\psi\left(\mathcal{M}_{2}\right)=\{(3,5),(2,5),(3,4),(1,4)\}\right)
\end{aligned}
$$

Let us note $\Delta: \mathbb{N}^{2} \rightarrow \mathbb{N}^{2}$ such that $\Delta(x, y)=(y, x)$. Another induction enables to prove that when $n \in\left[0,2^{r+1}-1\right],\left(s_{1,1}(r+1, n), s_{1,1}(r+1, n+1)\right)$ describes $\psi\left(\mathcal{M}_{r}\right) \cup \Delta\left(\psi\left(\mathcal{M}_{r}\right)\right)$. For $\ell \in \mathbb{N}^{*}$ and $m \in \mathcal{M}_{\ell}$, let $A_{r}(m)=\{\psi(m x) \mid x \in$ $\left.\mathcal{M}_{r}\right\} \cup\left\{\Delta(\psi(m x)) \mid x \in \mathcal{M}_{r}\right\}$. 
From now on, in order to simplify the notations, we will denote by $s(r, n)$ the value $s_{a, b}(r, n)$. Let us define the sequences $(S(r, n))_{r \in \mathbb{N}, n \in\left[0,2^{r}-1\right]}$ by $S(r, n)=(s(r, n), s(r, n+1))$ and $\left(S_{d}(r, n)\right)_{r \in \mathbb{N}, n \in\left[0,2^{r}-1\right]}$ by $S_{d}(r, n)=(s(r, n)$ $\bmod d, s(r, n+1) \bmod d)$. The following link between $\psi$ and $S$ can be proved by induction.

Lemma 1. Let $r \geqslant 0, \ell>0$ and let $m \in \mathcal{M}_{\ell}$ such that $\psi(m)=(a, b)$. Then $S(r+1,$.$) is a one to one map from \left[0,2^{r+1}-1\right]$ onto $A_{r}(m)$.

It means that in our case, the values $\psi(c)$ and $\Delta(\psi(c))$ for $c \in \mathcal{M}_{\ell}^{0}$ correspond to the elements $S(\ell+1, n)$ for $n \in\left[0,2^{\ell+1}-1\right]$ and $(a, b)=\left(F_{\ell+2}, F_{\ell+3}\right)$. Recently, Reznick proved in [35] that, for $d \geqslant 2,(a, b)=(1,1)$, and $r$ sufficiently large, the sequence $\left\{S_{d}(r, n)\right\}_{n \in \mathbb{N}}$ is well distributed among $\mathcal{S}_{d}:=\{(i \bmod d, j$ $\bmod d) \mid \operatorname{gcd}(i, j, d)=1\}$. We need a similar result for any couple $(a, b)$ in order to show that the values $\chi(c)$ are asymptotically well distributed modulo $d$. We will use similar notations and follow the arguments of [35] to prove the next theorem. We define :

- for $\gamma \in \mathcal{S}_{d}, B_{d}(r, \gamma):=\#\left\{n \in\left[0,2^{r}-1\right] \mid S_{d}(r, n)=\gamma\right\}$,

$-\chi_{d}$, the map such that $\chi_{d}(m)=\chi(m) \bmod d$,

$-\psi_{d}$ the map such that if $\psi(m)=(v, u)$ then $\psi_{d}(m)=(v \bmod d, u \bmod d)$,

$-N_{d}$ the cardinality of $\mathcal{S}_{d}$.

Theorem 3. Let $(a, b, d) \in \mathbb{N}^{3}$ such that $d$ be prime and $(a, d)=(b, d)=1$. There exist constants $c_{d}$ and $\rho_{d}<2$ so that if $m \in \mathbb{N}$ and $\alpha \in \mathcal{S}_{d}$, then for all $r \geqslant 0$,

$$
\left|B_{d}(r, \alpha)-\frac{2^{r}}{N_{d}}\right|<c_{d} \rho_{d}^{r}
$$

Proof. Due to the lack of space, we just give a short proof of this, following the arguments of section 4 in 35 and pointing out the differences. Since $d$ is prime, we have $\mathcal{S}_{d}=\mathbb{Z} / d \mathbb{Z} \times \mathbb{Z} / d \mathbb{Z} \backslash\{(0,0)\}$, and $N_{d}=d^{2}-1$. We can define a graph $\mathcal{G}_{d}$ and the applications $L$ and $R$ in the same way, and also have $L^{d}=R^{d}=\mathrm{id}$. In the proof of lemma 14, we have to give a slightly different proof that for each $\alpha=(x, y) \in \mathcal{S}_{d}$ there exists a way from $(a, b)$ to $\alpha$ in the graph $\mathcal{G}_{d}$. Notice that since $(0,0) \notin \mathcal{S}_{d}$, then either $x \neq 0$ or $y \neq 0$. If $y \neq 0$, we notice that $R^{k^{\prime}}\left(L^{k}(a, b)\right)=$ $\left(a+k^{\prime}(b+k a), b+k a\right)$. As $(a, d)=1$, we can choose $k$ such that $b+k a=y$. Thus, as $y \neq 0$, we can choose $k^{\prime}$ such that $a+k^{\prime}(b+k a)=x$ and we are done. If $x \neq 0$, then we consider $L^{k^{\prime}}\left(R^{k}(a, b)\right)=\left(a+k b, b+k^{\prime}(a+k b)\right):$ in the same way, we can choose $\left(k, k^{\prime}\right)$ such that $a+k b=x$ and $b+k^{\prime}(a+k b)=y$. In the first line of the proof of lemma 14 , we also have to consider $\left(r_{0}, n_{0}\right) \in \mathbb{N} \times \mathbb{N}$ such that $S_{d}\left(r_{0}, n_{0}\right)=(0,1)$ rather than $S_{d}(0,0)=(0,1)$. Thus the adjacency matrix of the graph satisfies the same properties as in theorem 15 of [35]. So the conclusion remains true for $B_{d}$.

From Theorem 3 and Lemma 1 we can now give the proof of Theorem 1. 
Proof. Let $\alpha \in(\mathbb{Z} / d \mathbb{Z})^{*}$, and $\left(\beta_{i}, \gamma_{i}\right)_{1 \leqslant i \leqslant d}$ the $d$ elements of $\mathbb{Z} / d \mathbb{Z} \times \mathbb{Z} / d \mathbb{Z} \backslash$ $\{(0,0)\}$ such that $\beta_{i}+\gamma_{i}=\alpha$, then

$$
\#\left\{x \in \mathcal{M}_{r} \mid \chi_{d}(m x)=\alpha\right\}=\sum_{i=1}^{d} \#\left\{x \in \mathcal{M}_{r} \mid \psi_{d}(m x)=\left(\beta_{i}, \gamma_{i}\right)\right\} .
$$

If $n \in\left[0,2^{r+1}\right]$ is such that $S_{d}(r+1, n)=\left(\beta_{i}, \gamma_{i}\right)$ then , thanks to Lemma 1 . it corresponds to $x \in \mathcal{M}_{r}$ such that $\psi_{d}(m x)=\left(\beta_{i}, \gamma_{i}\right)$ or $\psi_{d}(m x)=\left(\gamma_{i}, \beta_{i}\right)$. In this last case, there exists an integer $j$, such that $\left(\gamma_{i}, \beta_{i}\right)=\left(\beta_{j}, \gamma_{j}\right)$. Hence,

$$
\sum_{i=1}^{d} \#\left\{n \in\left[0,2^{r+1}-1\right] \mid S_{d}(r+1, n)=\left(\beta_{i}, \gamma_{i}\right)\right\}=2 \times \#\left\{x \in \mathcal{M}_{r} \mid \chi_{d}(m x)=\alpha\right\} .
$$

Now by definition of $B_{d}$ :

$$
\sum_{i=1}^{d} \#\left\{n \in\left[0,2^{r+1}-1\right] \mid S_{d}(r+1, n)=\left(\beta_{i}, \gamma_{i}\right)\right\}=\sum_{i=1}^{d} B_{d}\left(r+1,\left(\beta_{i}, \gamma_{i}\right)\right) .
$$

Thus,

$$
\frac{\#\left\{x \in \mathcal{M}_{r} \mid \chi_{d}(m x)=\alpha\right\}}{2^{r}}-\frac{d}{d^{2}-1}=\sum_{i=1}^{d}\left(\frac{B_{d}\left(r+1,\left(\beta_{i}, \gamma_{i}\right)\right)}{2^{r+1}}-\frac{1}{d^{2}-1}\right) .
$$

Then we can use Theorem 3 and the triangular inequality to prove the first inequality of the theorem. Using this inequality for $\alpha \neq 0$ we prove the second inequality.

\section{Proof of Theorem 2}

From now on, we will denote by $\bar{m}$, the value $\chi(m)$. Let us set $M=\sup \{\bar{m} \mid m \in$ $\left.\mathcal{M}_{\ell, p}\right\}$ and $I=\inf \left\{\bar{m} \mid m \in \mathcal{M}_{\ell, p}\right\}$. If $m \in \mathcal{M}_{\ell, p}$ is not one of the words of the points ii) (resp. iii)), we will propose $m^{\prime} \in \mathcal{M}_{\ell, p}$ such that $\overline{m^{\prime}}<\bar{m}$ (resp. $\overline{m^{\prime}}>\bar{m}$ ). The lemmas in the two following subsections give the details about the words $m^{\prime}$ we use to compare.

We first look for $m \in \mathcal{M}_{\ell, p}$ such that $\bar{m}=I$. First suppose that two 1's in the word $m$ are separated by one 0 or more. Then we can consider $(m, n, s) \in\left(\mathbb{N}^{*}\right)^{3}$ and $(a, b) \in \mathbb{N}^{2}$ and $(x, y) \in \mathcal{M}_{a} \times \mathcal{M}_{b}$ such that $m$ is one of the words

$$
1^{m} 0^{n} 1^{s}, \quad x 10^{m} 1^{n} 0 y \quad \text { or } \quad y 01^{n} 0^{m} 1 x .
$$

We won't consider the third case because it is the symmetric of the second one. The lemma 2 shows that $\overline{1^{m} 0^{n} 1^{s}}>\overline{1^{m+s} 0^{n}}$ and that $\overline{x 10^{m} 1^{n} 0 y}>\overline{x 1^{n+1} 0^{m+1} y}$. So if $\bar{m}=I$, there are no 0 between two 1 of the word $m$, and so there are integers $a$ and $c$ such that $m=0^{a} 1^{p} 0^{c}$. From lemma 3 we show that $a=0$ (and so $c=\ell-p$ ) or $c=0$ (and so $a=\ell-p$ ).

Now we look for $m \in \mathcal{M}_{\ell, p}$ such that $\bar{m}=M$. If there are two consecutive 1 's in the word $m$, as $2 p<\ell$ the word $m$ will also have two consecutive 0's. We can consider the symmetry such that a subword 00 appears in $m$ before a subword 11. In this case there exists $(a, b, n) \in \mathbb{N}^{3}$ and $(x, y) \in \mathcal{M}_{a} \times \mathcal{M}_{b}$ such 
that $m=x 00(10)^{n} 11 y$. In this case, we have from lemma 4 that $\bar{m}<\overline{x(01)^{n+2} y}$. Now assume that there are no subword 11 in $m$. If two 1's in the word $m$ are separated by two 0's or more, then there exists $(m, n, a, b) \in\left(\mathbb{N}^{*}\right)^{2} \times \mathbb{N}^{2}, x \in \mathcal{M}_{a}$ and $y \in \mathcal{M}_{b}$ such that $m$ is one of the words

$$
\begin{array}{ccc}
x 010^{n}(01)^{m}, \quad x 010^{n}(01)^{m} 0, & x 010^{n}(01)^{m} 0^{2} y, \\
10^{n}(01)^{m}, \quad 10^{n}(01)^{m} 0, \quad \text { or } \quad 10^{n}(01)^{m} 0^{2} y .
\end{array}
$$

The Lemmas (5) and (6) give us in any case $m^{\prime} \in \mathcal{M}_{\ell, p}$ such that $\bar{m}<\overline{m^{\prime}}$. We have just proved that 11 is not a subword of $m$, and that two 1's of $m$ are separated by exactly one 0 . So the word $m$ or its symmetric is $0^{a}(01)^{p} 0^{c}$ where $(a, c) \in \mathbb{N} \times \mathbb{N}$. The lemma (7) shows that $M$ is reached when $a=0$ (so $c=\ell-2 p$ ) or when $c=1$ (so $a=\ell-2 p-1$ ). Then the point iii) is proved.

Now we just have to apply Proposition 1 to deduce $i$ ) from ii) and iii).

\section{Lemmas to Find the Lower Bound}

Lemma 2. Let $(m, n, s) \in\left(\mathbb{N}^{*}\right)^{3}$ and $(a, b) \in \mathbb{N}^{2}$. Let $x \in \mathcal{M}_{a}$ and $y \in \mathcal{M}_{b}$. We have

$$
\text { i) } \frac{\overline{1^{m} 0^{n} 1^{s}}}{x 10^{m} 1^{n} 0 y}>\frac{\overline{1^{m+s} 0^{n}} \text { and }}{x 1^{n+1} 0^{m+1} y} \text {. }
$$

Proof. For the point i), we compute

$$
\begin{gathered}
\overline{1^{m} 0^{n} 1^{s}}=(1,2)\left(\begin{array}{cc}
1 & m \\
0 & 1
\end{array}\right)\left(\begin{array}{cc}
F_{n-1} & F_{n} \\
F_{n} & F_{n+1}
\end{array}\right)\left(\begin{array}{ll}
1 & s \\
0 & 1
\end{array}\right)\left(\begin{array}{l}
1 \\
1
\end{array}\right) \text {, so } \\
\overline{1^{m} 0^{n} 1^{s}}=(s+1) F_{n-1}+((s+1)(m+2)+1) F_{n}+(m+2) F_{n+1} . \\
\text { Also, } \overline{1^{m+s} 0^{n}}=(1,2)\left(\begin{array}{cc}
1 & m+s \\
0 & 1
\end{array}\right)\left(\begin{array}{cc}
F_{n-1} & F_{n} \\
F_{n} & F_{n+1}
\end{array}\right)\left(\begin{array}{l}
1 \\
1
\end{array}\right), \text { so } \\
\overline{1^{m+s} 0^{n}}=(2+m+s+1) F_{n+1}+(2+m+s) F_{n} .
\end{gathered}
$$

The difference between (21) and (3) is $m s F_{n}$, so it is positive in the conditions of the lemma. We can notice that there is equality when $s=0$ or when $m=0$, which can also be explained by the symmetry.

To prove the point $i i)$, let us set $(v, u)=\psi(x)$. We have

$$
\begin{gathered}
\psi\left(x 10^{m} 1^{n} 0\right)=(v, u)\left(\begin{array}{ll}
1 & 1 \\
0 & 1
\end{array}\right)\left(\begin{array}{cc}
F_{m-1} & F_{m} \\
F_{m} & F_{m+1}
\end{array}\right)\left(\begin{array}{ll}
1 & n \\
0 & 1
\end{array}\right)\left(\begin{array}{ll}
0 & 1 \\
1 & 1
\end{array}\right) \\
=\left(\left(n F_{m}+F_{m+1}\right) u+\left(n F_{m+1}+F_{m+2}\right) v, F_{m+2} u+\left(F_{m+1}+(n+1) F_{m+2}\right) v+(u-v) n F_{m}\right) .
\end{gathered}
$$

We also compute

$$
\psi\left(x 1^{n+1} 0^{m+1}\right)=(v, u)\left(\begin{array}{cc}
1 & n+1 \\
0 & 1
\end{array}\right)\left(\begin{array}{cc}
F_{m} & F_{m+1} \\
F_{m+1} & F_{m+2}
\end{array}\right)
$$




$$
=\left(F_{m+1} u+\left(F_{m}+(n+1) F_{m+1}\right) v, F_{m+2} u+\left(F_{m+1}+(n+1) F_{m+2}\right) v\right) .
$$

As $u>v$, we can deduce the point ii) from the comparison components by components of the vectors (4) and (5).

Lemma 3. Let $(a, b, c) \in\left(\mathbb{N}^{*}\right)^{3}$, we have $\overline{0^{a} 1^{b} 0^{c}}>\overline{1^{b} 0^{a+c}}$.

Proof. We first compute the left hand side

$$
\begin{gathered}
\overline{0^{a} 1^{b} 0^{c}}=(1,2)\left(\begin{array}{cc}
F_{a-1} & F_{a} \\
F_{a} & F_{a+1}
\end{array}\right)\left(\begin{array}{ll}
1 & b \\
0 & 1
\end{array}\right)\left(\begin{array}{cc}
F_{c-1} & F_{c} \\
F_{c} & F_{c+1}
\end{array}\right)\left(\begin{array}{l}
1 \\
1
\end{array}\right) \\
=\left(F_{a+2} F_{c-1}+\left(b F_{a+2}+F_{a+3}\right) F_{c}+F_{a+2} F_{c}+\left(b F_{a+2}+F_{a+3}\right) F_{c+1}+\right) \\
=F_{a+2} F_{c+1}+F_{a+3} F_{c+2}+b F_{a+2} F_{c+2} .
\end{gathered}
$$

We also compute the right hand side

$$
\begin{gathered}
\overline{1^{b} 0^{a+c}}=(1,2)\left(\begin{array}{ll}
1 & b \\
0 & 1
\end{array}\right)\left(\begin{array}{cc}
F_{a+c-1} & F_{a+c} \\
F_{a+c} & F_{a+c+1}
\end{array}\right)\left(\begin{array}{l}
1 \\
1
\end{array}\right) \\
=\left(F_{a+c-1}+(b+2) F_{a+c}+F_{a+c}+(b+2) F_{a+c+1}\right) \\
=F_{a+c+4}+b F_{a+c+2} .
\end{gathered}
$$

With eq. 1, page 242 we show that it is

$$
F_{a+2} F_{c+1}+F_{a+3} F_{c+2}+b F_{a+c+2} .
$$

The difference between (6) and (7) is $b\left(F_{a+c+2}-F_{a+2} F_{c+2}\right)=b F_{c} F_{a}$, which is positive in the conditions of the lemma. In the cases $c=0$ or $a=0$, there is equality which we already knew by the symmetry.

\section{Lemmas to Compute the Upper Bound}

Lemma 4. Let $(n, a, b) \in \mathbb{N}^{3}$. For all $x \in \mathcal{M}_{a}$ and $y \in \mathcal{M}_{b}$ we have

$$
\overline{x 00(10)^{n} 11 y}<\overline{x(01)^{n+2} y} \text {. }
$$

Proof. We first compute

$$
S_{0}^{2}\left(S_{1} S_{0}\right)^{n} S_{1}^{2}=\left(\begin{array}{ll}
1 & 1 \\
1 & 2
\end{array}\right)\left(\begin{array}{cc}
\alpha_{n} & 2 \beta_{n} \\
\beta_{n} & \alpha_{n}
\end{array}\right)\left(\begin{array}{ll}
1 & 2 \\
0 & 1
\end{array}\right)=\left(\begin{array}{cc}
\alpha_{n}+\beta_{n} & 3 \alpha_{n}+4 \beta_{n} \\
\alpha_{n}+2 \beta_{n} & 4 \alpha_{n}+6 \beta_{n}
\end{array}\right) .
$$

We set $(v, u)=\psi(x)$, so we have

$$
\begin{aligned}
\psi\left(x 00(10)^{n} 11\right) & =(v, u)\left(\begin{array}{cc}
\alpha_{n}+\beta_{n} & 3 \alpha_{n}+4 \beta_{n} \\
\alpha_{n}+2 \beta_{n} & 4 \alpha_{n}+6 \beta_{n}
\end{array}\right) \\
& =\left(v\left(\alpha_{n}+\beta_{n}\right)+u\left(\alpha_{n}+2 \beta_{n}\right), v\left(3 \alpha_{n}+4 \beta_{n}\right)+u\left(4 \alpha_{n}+6 \beta_{n}\right)\right) .
\end{aligned}
$$


From the other hand

$$
\begin{gathered}
S_{0} S_{1}\left(S_{0} S_{1}\right)^{n} S_{0} S_{1}=\left(\begin{array}{ll}
0 & 1 \\
1 & 2
\end{array}\right)\left(\begin{array}{cc}
\alpha_{n}-\beta_{n} & \beta_{n} \\
\beta_{n} & \alpha_{n}+\beta_{n}
\end{array}\right)\left(\begin{array}{ll}
0 & 1 \\
1 & 2
\end{array}\right) \\
=\left(\begin{array}{c}
\alpha_{n}+\beta_{n} \\
2 \alpha_{n}+3 \beta_{n} \\
2 \alpha_{n}+3 \beta_{n}+7 \beta_{n}
\end{array}\right), \text { so } \\
\psi\left(x(01)^{n+2}\right)=\left(v\left(\alpha_{n}+\beta_{n}\right)+u\left(2 \alpha_{n}+3 \beta_{n}\right), v\left(2 \alpha_{n}+3 \beta_{n}\right)+u\left(5 \alpha_{n}+7 \beta_{n}\right)\right) .
\end{gathered}
$$

As $v<u$ we can compare the vectors (8) and (9) components by components and then conclude.

Lemma 5. Let $(m, n, a, b) \in\left(\mathbb{N}^{*}\right)^{2} \times \mathbb{N}^{2}$. For all $x \in \mathcal{M}_{a}$ and $y \in \mathcal{M}_{b}$, we have

$$
\begin{aligned}
& \text { i) } \frac{\overline{x 010^{n}(01)^{m}}}{\text { ii) }}<\frac{\overline{x\left(010^{n}(01)^{m} 0\right.}}{\text { iii) }}<\frac{\overline{x(01)^{m+1} 0^{n+1}}}{x 010^{n}(01)^{m} 0^{2} y}<\frac{<(01)^{m+1} 0^{n+2} y}{}
\end{aligned}
$$

Proof. We compute

$$
S_{0} S_{1} S_{0}^{n}=\left(\begin{array}{ll}
0 & 1 \\
1 & 2
\end{array}\right)\left(\begin{array}{cc}
F_{n-1} & F_{n} \\
F_{n} & F_{n+1}
\end{array}\right)=\left(\begin{array}{cc}
F_{n} & F_{n+1} \\
F_{n+2} & F_{n+3}
\end{array}\right) .
$$

We deduce

$$
\begin{aligned}
S_{0} S_{1} S_{0}^{n}\left(S_{0} S_{1}\right)^{m} & =\left(\begin{array}{cc}
F_{n} & F_{n+1} \\
F_{n+2} & F_{n+3}
\end{array}\right)\left(\begin{array}{cc}
\alpha_{m}-\beta_{m} & \beta_{m} \\
\beta_{m} & \alpha_{m}+\beta_{m}
\end{array}\right) \quad \text { and so } \\
S_{0} S_{1} S_{0}^{n}\left(S_{0} S_{1}\right)^{m} & =\left(\begin{array}{cc}
\alpha_{m} F_{n}+\beta_{m} F_{n-1} & \alpha_{m} F_{n+1}+\beta_{m} F_{n+2} \\
\alpha_{m} F_{n+2}+\beta_{m} F_{n+1} & \alpha_{m} F_{n+3}+\beta_{m} F_{n+4}
\end{array}\right) .
\end{aligned}
$$

From the other hand, we can write $\left(S_{0} S_{1}\right)^{m+1} S_{0}^{n}=\left(S_{0} S_{1}\right)^{m} S_{0} S_{1} S_{0}^{n}$ so

$$
\begin{gathered}
\left(S_{0} S_{1}\right)^{m+1} S_{0}^{n}=\left(\begin{array}{cc}
\alpha_{m}-\beta_{m} & \beta_{m} \\
\beta_{m} & \alpha_{m}+\beta_{m}
\end{array}\right)\left(\begin{array}{cc}
F_{n} & F_{n+1} \\
F_{n+2} & F_{n+3}
\end{array}\right) \text {, and then } \\
\left(S_{0} S_{1}\right)^{m+1} S_{0}^{n}=\left(\begin{array}{cc}
\alpha_{m} F_{n}+\beta_{m} F_{n+1} & \alpha_{m} F_{n+1}+\beta_{m} F_{n+2} \\
\alpha_{m} F_{n+2}+\beta_{m}\left(F_{n}+F_{n+2}\right) & \alpha_{m} F_{n+3}+\beta_{m}\left(F_{n+1}+F_{n+3}\right)
\end{array}\right)
\end{gathered}
$$

Let us set $(v, u)=\psi(x)$.

$$
\begin{aligned}
& \psi\left(x 010^{n}(01)^{m}\right)=(v, u)\left(\begin{array}{cc}
\alpha_{m} F_{n}+\beta_{m} F_{n-1} & \alpha_{m} F_{n+1}+\beta_{m} F_{n+2} \\
\alpha_{m} F_{n+2}+\beta_{m} F_{n+1} & \alpha_{m} F_{n+3}+\beta_{m} F_{n+4}
\end{array}\right)\left(\begin{array}{l}
1 \\
1
\end{array}\right) \\
= & v\left(\alpha_{m} F_{n+2}+\beta_{m} F_{n-1}+\beta_{m} F_{n+2}\right)+u\left(\alpha_{m} F_{n+4}+\beta_{m} F_{n+1}+\beta_{m} F_{n+4}\right) .
\end{aligned}
$$

We also have

$$
\psi\left(x(01)^{m+1} 0^{n}\right)=(v, u)\left(\begin{array}{cc}
\alpha_{m} F_{n}+\beta_{m} F_{n+1} & \alpha_{m} F_{n+1}+\beta_{m} F_{n+2} \\
\alpha_{m} F_{n+2}+\beta_{m}\left(F_{n}+F_{n+2}\right) & \alpha_{m} F_{n+3}+\beta_{m}\left(F_{n+1}+F_{n+3}\right)
\end{array}\right)\left(\begin{array}{l}
1 \\
1
\end{array}\right)
$$




$$
=v\left(\alpha_{m} F_{n+2}+\beta_{m} F_{n+1}+\beta_{m} F_{n+2}\right)+u\left(\alpha_{m} F_{n+4}+\beta_{m} F_{n+2}+\beta_{m} F_{n+4}\right) .
$$

The difference between (13) and (12) is $v \beta_{m} F_{n}+u \beta_{m} F_{n}$ so we deduce the first point.

To prove the point ii) we use (10) which gives

$$
S_{0} S_{1} S_{0}^{n}\left(S_{0} S_{1}\right)^{m} S_{0}=\left(\begin{array}{cc}
\alpha_{m} F_{n+1}+\beta_{m} F_{n+2} & \alpha_{m} F_{n+2}+\beta_{m} F_{n-1}+\beta_{m} F_{n+2} \\
\alpha_{m} F_{n+3}+\beta_{m} F_{n+4} & \alpha_{m} F_{n+4}+\beta_{m} F_{n+1}+\beta_{m} F_{n+4}
\end{array}\right)
$$

Let us set $(v, u)=\psi(x)$. We have

$$
\begin{aligned}
\overline{x 010^{n}(01)^{m} 0}= & v\left(\alpha_{m} F_{n+3}+\beta_{m} F_{n-1}+2 \beta_{m} F_{n+2}\right) \\
& +u\left(\alpha_{m} F_{n+5}+\beta_{m} F_{n+1}+2 \beta_{m} F_{n+4}\right) .
\end{aligned}
$$

From (11) we deduce

$$
\left(S_{0} S_{1}\right)^{m+1} S_{0}^{n+1}=\left(\begin{array}{cc}
\alpha_{m} F_{n+1}+\beta_{m} F_{n+2} & \alpha_{m} F_{n+2}+\beta_{m} F_{n+3} \\
\alpha_{m} F_{n+3}+\beta_{m} F_{n+1}+\beta_{m} F_{n+3} & \alpha_{m} F_{n+4}+\beta_{m} F_{n+2}+\beta_{m} F_{n+4}
\end{array}\right)
$$

So

$$
\overline{x(01)^{m+1} 0^{n+1}}=v\left(\alpha_{m} F_{n+3}+\beta_{m} F_{n+4}\right)+u\left(\alpha_{m} F_{n+5}+\beta_{m} F_{n+3}+\beta_{m} F_{n+5}\right) .
$$

The difference between (17) and (15) is $v \beta_{m} F_{n}$ so we have the positivity. To prove iii) we compute from (14) and (16)

$$
S_{0} S_{1} S_{0}^{n}\left(S_{0} S_{1}\right)^{m} S_{0}^{2}=\left(\begin{array}{c}
\alpha_{m} F_{n+2}+\beta_{m} F_{n-1}+\beta_{m} F_{n+2} \alpha_{m} F_{n+3}+\beta_{m} F_{n-1}+2 \beta_{m} F_{n+2} \\
\alpha_{m} F_{n+4}+\beta_{m} F_{n+1}+\beta_{m} F_{n+4} \alpha_{m} F_{n+5}+\beta_{m} F_{n+1}+2 \beta_{m} F_{n+4}
\end{array}\right)
$$

and $\left(S_{0} S_{1}\right)^{m+1} S_{0}^{n+2}=\left(\begin{array}{cc}\alpha_{m} F_{n+2}+\beta_{m} F_{n+3} & \alpha_{m} F_{n+3}+\beta_{m} F_{n+4} \\ \alpha_{m} F_{n+4}+\beta_{m} F_{n+2}+\beta_{m} F_{n+4} & \alpha_{m} F_{n+5}+\beta_{m} F_{n+3}+\beta_{m} F_{n+5}\end{array}\right)$.

We compare components by components and then deduce iii)

Lemma 6. Let $(m, n, b) \in\left(\mathbb{N}^{*}\right)^{2} \times \mathbb{N}$ and $y \in \mathcal{M}_{b}$. We have

$$
\begin{aligned}
& \text { i) } \overline{10^{n}(01)^{m}}<\overline{(01)^{m+1} 0^{n-1}} \text {, } \\
& \text { ii) } \overline{10^{n}(01)^{m} 0}<\overline{(01)^{m+1} 0^{n}} \\
& \text { iii) } \overline{10^{n}(01)^{m} 0^{2} y}<\overline{(01)^{m+1} 0^{n+1} y} \text {. }
\end{aligned}
$$

Proof. We will use the computations of the proof of lemma 5. Let us consider that $\psi(x)=(1,1)$. In this case $\psi(x 0)$ would be $(1,2)$, and we would have $\overline{x 010^{n}(01)^{m}}=\overline{10^{n}(01)^{m}}$. So with (12) we have

$$
\overline{10^{n}(01)^{m}}=\alpha_{m} F_{n+2}+\beta_{m} F_{n-1}+\beta_{m} F_{n+2}+\alpha_{m} F_{n+4}+\beta_{m} F_{n+1}+\beta_{m} F_{n+4} .
$$


With (11) we have

$\overline{(01)^{m+1} 0^{n-1}}=\alpha_{m} F_{n+1}+\beta_{m} F_{n}+\beta_{m} F_{n+1}+2 \alpha_{m} F_{n+3}+2 \beta_{m} F_{n+1}+2 \beta_{m} F_{n+3}$,

so $\overline{(01)^{m+1} 0^{n-1}}-\overline{10^{n}(01)^{m}}=\alpha_{m} F_{n-1}+\beta_{m} F_{n+2}$, and then we have the point i). In the same way, we also have

$\overline{10^{n}(01)^{m} 0}=\alpha_{m} F_{n+3}+\beta_{m} F_{n-1}+2 \beta_{m} F_{n+2}+\alpha_{m} F_{n+5}+\beta_{m} F_{n+1}+2 \beta_{m} F_{n+4}$, and $\overline{(01)^{m+1} 0^{n}}=\alpha_{m} F_{n+2}+\beta_{m} F_{n+1}+\beta_{m} F_{n+2}+2 \alpha_{m} F_{n+4}+\beta_{m} F_{n+2}+\beta_{m} F_{n+4}$, so $\overline{(01)^{m+1} 0^{n}}-\overline{10^{n}(01)^{m} 0}=F_{n}\left(\alpha_{m}+2 \beta_{m}\right)$ and then we deduce the point $\left.i i\right)$.

Now, $\psi\left(10^{n}(01)^{m} 0^{2}\right)=(1,1) S_{0} S_{1} S_{0}^{n}\left(S_{0} S_{1}\right)^{m} S_{0}^{2}$, and with (15) we find

$$
\begin{aligned}
\psi\left(10^{n}(01)^{m} 0^{2}\right)=\left(\alpha_{m}\left(F_{n+2}+F_{n+4}\right)\right. & +\beta_{m}\left(F_{n-1}+F_{n+5}\right), \\
& \left.\alpha_{m}\left(F_{n+3}+F_{n+5}\right)+\beta_{m}\left(F_{n-1}+F_{n+5}\right)\right) .
\end{aligned}
$$

With (11) we compute

$$
\begin{aligned}
\psi\left((01)^{m+1} 0^{n+1}\right)=\left(\alpha _ { m } \left(F_{n+1}+\right.\right. & \left.2 F_{n+3}\right)+\beta_{m}\left(F_{n+1}+3 F_{n+3}\right), \\
& \alpha_{m}\left(F_{n+2}+2 F_{n+4}\right)+\beta_{m}\left(F_{n+2}+3 F_{n+4}\right) .
\end{aligned}
$$

The difference of the first component of (21) by the first component of (20) is $\alpha_{m} F_{n-2}+\beta_{m} F_{n+2}$, and the difference of the second components is $\alpha_{m} F_{n}+$ $\beta_{m}\left(2 F_{n+4}-F_{n-1}-F_{n+1}\right)$ so the point iii) is proved.

Lemma 7. Let $(a, b, c) \in \mathbb{N} \times \mathbb{N}^{*} \times \mathbb{N}$. If $c \neq 1$ and $a \neq 0$ then $\overline{0^{a}(01)^{b} 0^{c}}<$ $\overline{(01)^{b} 0^{a+c}}$.

Proof. We compute

$$
\begin{gathered}
\overline{0^{a}(01)^{b} 0^{c}}=(1,2)\left(\begin{array}{cc}
F_{a-1} & F_{a} \\
F_{a} & F_{a+1}
\end{array}\right)\left(\begin{array}{cc}
\alpha_{b}-\beta_{b} & \beta_{b} \\
\beta_{b} & \alpha_{b}+\beta_{b}
\end{array}\right)\left(\begin{array}{cc}
F_{c-1} & F_{c} \\
F_{c} & F_{c+1}
\end{array}\right)\left(\begin{array}{l}
1 \\
1
\end{array}\right) \\
=\alpha_{b}\left(F_{a+2} F_{c+1}+F_{a+3} F_{c+2}\right)+\beta_{b}\left(F_{a+1} F_{c+1}+F_{a+4} F_{c+2}\right) .
\end{gathered}
$$

On the other hand

$$
\begin{gathered}
\overline{(01)^{b} 0^{a+c}}=(1,2)\left(\begin{array}{cc}
\alpha_{b}-\beta_{b} & \beta_{b} \\
\beta_{b} & \alpha_{b}+\beta_{b}
\end{array}\right)\left(\begin{array}{cc}
F_{a+c-1} & F_{a+c} \\
F_{a+c} & F_{a+c+1}
\end{array}\right)\left(\begin{array}{l}
1 \\
1
\end{array}\right) \\
=\alpha_{b} F_{a+c+4}+\beta_{b}\left(F_{a+c+2}+F_{a+c+4}\right) .
\end{gathered}
$$

Using eq. 11, we prove that the difference between (23) by (22) is $\beta_{b} F_{a}\left(F_{c+1}-F_{c}\right)$. It is zero if and only if $a=0$ or $c=1$, and positive otherwise. 